УДК 676.15/.16.054.1(075)

\title{
ПОСТРОЕНИЕ РИСУНКА ГАРНИТУРЫ С УДАРНЫМ ЭФФЕКТОМ В НОЖЕВЫХ РАЗМАЛЫВАЮЩИХ МАШИНАХ
}

\author{
() В.И. Ковалев", В.А. Кожухов, Ю.Д. Алашкевич \\ Сибирский государственный технологический университет, пр. Мира, 82, \\ Красноярск, 660049 (Россия), e-mail: mapt@sibstu.kts.ru
}

\begin{abstract}
Проведено построение и графо-аналитическая проверка исполнения рисунка с трапецеидальной формой рабочей поверхности единичных ножей гарнитуры ротора и статора на одновременность контакта по всей длине режущих их кромок, сходящихся в центре диска.

Выведены аналитические зависимости для определения времени прохождения различными точками режущих кромок ножей вращающегося ротора окружных дуг, соединяющих эти точки с соответствующими точками режущих кромок ножей неподвижного статора.

Ключевые слова: время, гарнитура, диск, единичный, исполнение, кромка, нож, размол, режущая, рисунок, ротор, статор, окружная скорость, конструктивный, параметры.
\end{abstract}

\section{Введение}

Целесообразность проверки основана на том, что при одновременном контакте режущих кромок ножей ротора и статора превалирует «ударный эффект», за счет чего на нависшие на них волокнистые наслойки оказывается значительное по величине силовое ударное воздействие. Этому способствует кратковременность контакта.

Хорошо известно, что по сравнению с протяженным во времени «эффектом ножниц» кратковременное ударное воздействие интенсифицирует процесс размола, повышает его эффективность и качество.

В связи с этим изучение особенностей ножевой геометрии, основанной на условии одновременности контакта по длине режущих кромок, представляет значительный интерес.

Можно предположить, что выполнение этого условия делает реальной замену «эффекта ножниц» на «ударный эффект».

Представляется, что это позволит:

- снизить долю поперечного резания волокон;

- увеличить долю фибрилляции волокон (в виде их раздавливания и продольного разделения).

Также представляется, что наиболее близким к «ударному эффекту» является авторское исполнение с трапецеидальной формой рабочей поверхности ножей ротора и статора [1-3]. Окончательный ответ на

Ковалев Валерий Иванович - доцент кафедры машин и аппаратов промышленных технологий, кандидат технических наук, тел.: (391) 227-34-53,

e-mail: mapt@sibstu.kts.ru

Кожухов Виктор Анатольевич - старший преподаватель кафедры машин и аппаратов промышленных технологий, тел.: (391) 227-34-53, e-mail: mapt@sibstu.kts.ru

Алашкевич Юрий Давыдович - заведующий кафедрой машин и аппаратов промышленных технологий МАПТ, профессор, доктор технических наук, тел.: (3912) 27-34-53, e-mail: mapt@ sibstu.kts.ru это предположение могут дать только результаты экспериментальной графо-аналитической проверки данного исполнения.

\section{Графо-аналитический эксперимент}

На рисунке представлена фронтальная проекция рисунка сопряжения ножевых поверхностей сцентрированных дисков ротора и статора, построенного в соответствии с особенностями трапецеидального исполнения [1-3].

\footnotetext{
* Автор, с которым вести переписку.
} 


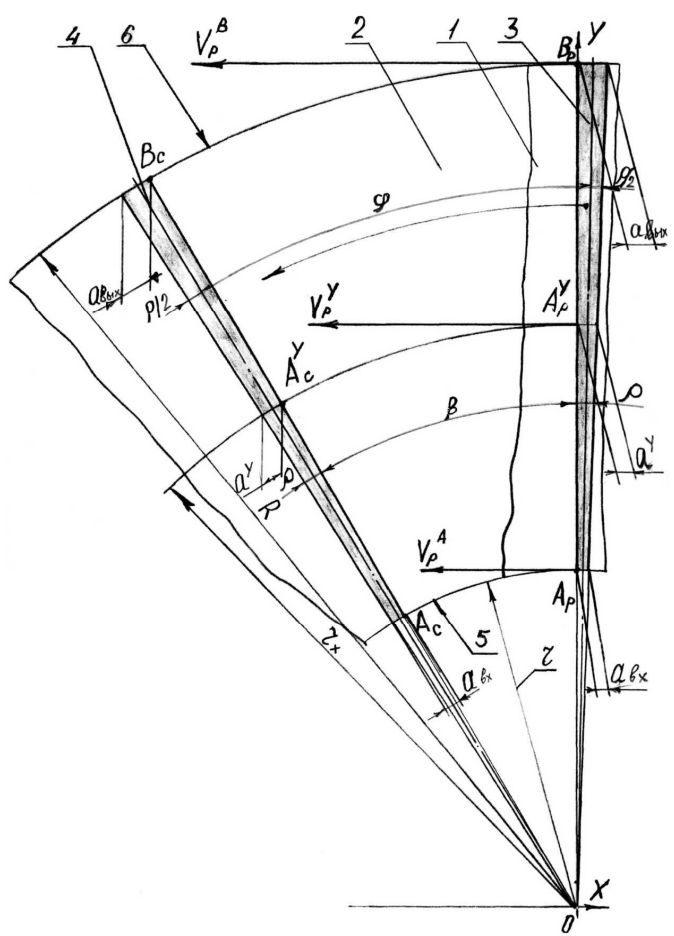

Фронтальная проекция рисунка сопряжения ножевых поверхностей сцентрированных дисков ротора и статора: 1 - ножевая поверхность ротора; 2 - ножевая поверхность статора; 3 - единичный нож ротора; 4 - единичный нож статора; 5 - входная окружная кромка сопряженных дисков; 6 - выходная окружная кромка
Введем следующие обозначения:r - радиус входной окружных кромки 5 и дуги $A_{P} A_{C} ; r_{Y}$ - радиус произвольной окружной дуги $A_{P}^{Y} A_{C}^{Y} ; R-$ радиус выходной окружных кромки 6 и дуги $B_{P} B_{C} ; A_{P} B_{P}$ - режущая кромка единичного ножа 3 ротора; $A_{C} B_{C}-$ режущая кромка единичного ножа 4 статора; а - толщина единичных ножей ротора и статора; $\rho$ - угол между кромками, ограничивающими рабочую поверхность единичных ножей; $\beta$ угол, на который необходимо повернуть кромку $A_{P} B_{P}$, чтобы совместить лежащие на ней точки $A_{P}, A_{P}^{Y}$ и $B_{P}$ с точками $A_{C}, A_{C}^{Y} \quad{ }^{\prime} B_{C} \quad A_{C}$, лежащими на кромке $(\beta=\varphi-\rho) ; \varphi-$ угол между осями симметрии ножей 3 и $4 ; a_{b x}$ - толщина единичного ножа на входной окружной кромке $5 ; a^{Y}$ - толщина единичного ножа на окружной кромке $A_{P}^{Y} A_{C}^{Y} ; a_{\text {выx }}$ - толщина единичного ножа на выходной окружной кромке 6.

Окружной стрелкой показано направление вращения ротора.

Время прохождения произвольной точкой $A_{P}^{Y}$ окружной дуги $\widehat{A}_{P}^{Y} \widehat{A}_{C}^{Y}$

Согласно рисунку угол $\beta$ имеет постоянное значение на всей длине режущих кромок $A_{P} B_{P}$ и $A_{C} B_{C}$.

Определяется по формуле

$$
t^{A_{P}^{Y}}=\frac{\widehat{A}_{P}^{Y} \widehat{A}_{C}^{Y}}{V_{O}^{A_{P}^{Y}}}
$$

Длина дуги определяется по формуле $\widehat{A}_{P}^{Y} \widehat{A}_{C}^{Y}=\frac{c_{Y} \cdot \beta}{360^{\circ}}$.

Длина окружности $c_{Y}$ определяется по формуле

$$
c_{Y}=2 \cdot \pi \cdot r_{y}
$$

После подстановки (3) в (2), получим

$$
\begin{aligned}
& \widehat{A}_{P}^{Y} \widehat{A}_{C}^{Y}=\frac{2 \cdot \pi \cdot r_{Y} \cdot \beta}{360^{\circ}} . \\
& V_{O}^{A_{P}^{Y}}=\frac{V_{O}^{A_{P}} \cdot r_{Y}}{r} .
\end{aligned}
$$

Рисунок сопряжения трапецеидальных поверхностей единичных ножей дисков ротора и статора [1-3]. $V_{O}^{A_{P}}$ - окружная скорость в точке $A_{P}-V_{O}^{A_{P}}=2 \cdot \pi \cdot r \cdot n$.

После подстановки в (5), согласно зависимости $V_{O}^{A_{P}^{Y}}=2 \cdot \pi \cdot r_{Y} \cdot n$

После подстановки (4) и (6) в (1), получим

$$
t^{A_{P}^{Y}}=\frac{\beta}{360^{\circ} \cdot n} \text {. }
$$

Согласно рисунку

$$
\beta=\varphi-\rho \text {. }
$$


После подстановки в (7), получим окончательно

$$
t^{A_{P}^{Y}}=\frac{\varphi-\rho}{360^{\circ} \cdot n}
$$

Время прохождения точкой $A_{P}$ окружной дуги $\widehat{A}_{P} \widehat{A}_{C}$.

Определяется по формуле (1) $\quad t^{A_{P}}=\frac{\widehat{A}_{P} \widehat{A}_{C}}{V_{O}^{A_{P}}}$.

Длина дуги

$$
\widehat{A}_{P} \widehat{A}_{C}=\frac{2 \cdot \pi \cdot r \cdot \beta}{360^{\circ}} \text {. }
$$

Окончательно

$$
t^{A_{P}}=\frac{\beta}{360 \cdot n}=\frac{\varphi-\rho}{360^{\circ} \cdot n} .
$$

Время прохождения произвольной точкой $B_{P}$ окружной дуги $\widehat{B}_{P} \widehat{B}_{C}$

Определяется по формуле (1) $\quad t^{B_{P}}=\frac{\widehat{B}_{P} \widehat{B}_{C}}{V_{O}^{B_{P}}}$.

Длина дуги

$$
\widehat{B}_{P} \widehat{B}_{C}=\frac{2 \cdot \pi \cdot R \cdot \beta}{360^{\circ}}
$$

Окружная скорость $V_{O}^{B_{P}}$ в точке $B_{P} \quad V_{O}^{B_{P}}=\frac{V_{O}^{A_{P}} \cdot R}{r}=2 \cdot \pi \cdot R \cdot n$.

Окончательно

$$
t^{B_{P}}=\frac{\beta}{360 \cdot n}=\frac{\varphi-\rho}{360^{\circ} n} \text {. }
$$

\section{Обсужнение результатов}

Результаты графо-аналитического эксперимента показывают, что при трапецеидальном исполнении размалывающих поверхностей единичных ножей ротора и статора [1-3]:

- время прохождения точками $A_{P}, A_{P}^{Y}$ и $B_{P}$ окружных дуг, соответственно $\widehat{A}_{P} \widehat{A}_{C}, \widehat{A}_{P}^{Y} \widehat{A}_{C}^{Y}$ и $\widehat{B}_{P} \widehat{B}_{C}$, одинаково;

- контакт режущих кромок ножей ротора и статора происходит по всей их длине.

\section{Вblводы}

Выявлено трапецеидальное исполнение рисунка рабочей поверхности единичных ножей ротора и статора.

Учитывая кратковременность ударного контакта, можно предположить, что это обеспечит:

- значительное по величине силовое ударное воздействие, способствующее разделению волокнистых наслоек и самих волокон по наиболее слабым продольным межволоконным связям;

- увеличению доли фибрилляции;

- снижение доли резки волокон по их поперечному сечению за счет сокращения «эффекта ножниц».

\section{Список литературы}

1. Патент 2227826 (RU). Размалывающая гарнитура для дисковой мельницы / Ю.Д. Алашкевич, В.И. Ковалев. 2004.

2. Ковалев В.И., Алашкевич Ю.Д., Набиева А.А. Влияние рисунка гарнитуры на процесс размола волокнистых полуфабрикатов. Красноярск, 2010. Ч. 1. 168 с.

3. Кожухов В.А., Ковалев В.И., Алашкевич Ю.Д. Ударный эффект при воздействии ножевой гарнитуры как фактор повышения качества размола // Вестник Сибирского государственного аэрокосмического университета имени академика М.Ф. Решетнева. 2006. №5. С. 130-132. 
Kovalev V.I. ${ }^{*}$, Kozhukhov V.A., Alashkevich Iu.D. CONSTRUCTION DRAWING SETS WITH THE SHOCK EFFECT OF THE MILLING CUTTER MACHINES

Siberian State Technological University, pr. Mira, 82, Krasnoyarsk, 660049 (Russia), e-mail: mapt@sibstu.kts.ru

The article held to build and grapho-analytical checking of drawings with the trapezoidal shape of the working surface of single headset rotor and stator knives on the simultaneity of contact along the entire length of the cutting edge of converging in the Centre of the disc. Withdrawn analytical dependences for determination of time passing various points of cutting edges of the blades rotating rotor district arcs connecting these dots with corresponding points on the cutting edges of the blades stationary stator. A discussion of the results. According to the results of the Graf-analytical experiment, conclusions are made.

Keywords: Time, headset, disc, single, perform, edge, knife, grinding, cutting, drawing, rotor, stator, peripheral speed, constructive options.

\section{References}

1. Patent 2227826 (RU). 2004. (in Russ.).

2. Kovalev V.I., Alashkevich Iu.D, Nabieva A.A. Vliianie risunka garnitury na protsess razmola voloknistykh polufabrikatov. [Effect of drawing on the headset grinding process fibrous semifinished]. Krasnoyarsk, 2010, part. 1, 168 p. (in Russ.).

3. Kozhukhov V.A., Kovalev V.I., Alashkevich Iu.D. Vestnik Sibirskogo gosudarstvennogo aerokosmicheskogo universiteta imeni akademika M.F. Reshetneva, 2006, no. 5, pp. 130-132. (in Russ.).

Received February 15, 2013

\footnotetext{
"Corresponding author.
} 